\title{
Information System Domain Modeling for Adaptive Learning
}

\author{
Anqi Chen and Jimei $\mathrm{Li}^{*}$ \\ College of Information Sciences, Beijing Language and Culture University, China \\ *Corresponding author
}

\begin{abstract}
To meet the need of the adaptive learning platform, the relevant research results of the domain modeling have been analyzed and compared in this paper, including the methods of domain knowledge representation, the domain modeling methods and modeling technology based on ontology. And combining with the characteristics of the information system domain knowledge, the aforementioned modeling methods and modeling technology have been compared and evaluated respectively.
\end{abstract}

Keywords-adaptive learning; information system; domain knowledge; knowledge modeling; ontology representation

\section{INTRODUCTION}

This Presently, the knowledge modeling is one of the most popular research fields and it ranges over a number of areas. But in the field of information studies, there is little research especially about the information system domain modeling teaching oriented on teaching. However, with the rise of online learning mode such as Moodle system. a learning platform highly efficient and applicable to each learner has become the urgent needs. On this platform, learners study by their own on the basis of knowledge accordingly. Thus in the process of building such a platform, knowledge modeling has become an essential cornerstone, and the information system, of course, is no exception. At the same time, there are no uniform standards for the professional training program in the field of information system in domestic universities, Information system knowledge modeling will also be an important supplement to this defect.

Knowledge is the familiarity of a certain object for people and acquired acquaintance, judgment or skills by learning, practicing and exploring. It includes the fact, information description, or skills acquired in education and practice. Knowledge is a unit of knowledge point and a proper representation and description of knowledge points. Literature[1,2] considers that knowledge points are mainly divided into two categories. One is the meta knowledge point, which means the smallest unit of knowledge that can not be cut. Another is the compound knowledge point, which are formed by the combination of the meta knowledge points. But in the real world the knowledge is not so straightforward and easy. Literature[3,4] mentioned there is a need for complicated knowledge from different knowledge to be structured and standardized to achieve a better understanding of knowledge, and this is what we called knowledge modeling. Literature[3] points out that knowledge is hierarchical and can be divided into four levels: the fact, the concept, the rule and the heuristic. It introduced the 3D knowledge space proposed by Heyes-Roth and widely used. And on the basis of the knowledge classification, it also proposed the knowledge representation and knowledge model, which is the result of knowledge modeling.

The knowledge model mainly includes three parts: domain knowledge, reasoning knowledge and task knowledge. The first part is domain knowledge, which mainly deals with the knowledge level of the first three levels (facts, concepts, rules) of information, that is, a detailed description of the specific knowledge and information types in a particular field. It mainly includes the field types, field facts and domain rules. Domain knowledge modeling is an effective method of knowledge management. Literature[3] mentioned construction of knowledge model is a basic and important process in the development of knowledge system. It can reflect the main function of the knowledge system. and help developers to sort out the knowledge system and effectively identify the key knowledge, so as to reduce unnecessary duplication of knowledge acquisition work.

It is considered that domain knowledge modeling is very meaningful for adaptive learning system. Because in the adaptive learning system, the system will develop the corresponding learning programs according to the learning progress and ability of the individuals. The key lies in providing different ways of presenting different learners in the field of knowledge. And the foundation to accomplish this function is a good domain knowledge model.

Knowledge representation is one of the key technologies of domain knowledge modeling. Literature[5,6] points out that the knowledge modeling based on ontology is a more effective and mature method of knowledge modeling. As literature [4] mentions, the main task of ontology knowledge modeling is to describe the objective concept and relationship. The main advantage of the ontology is that it can make domain knowledge standardized, and achieve effective knowledge sharing.

Up to the present study, according to the result of searching China National Knowledge Infrastructure database, there are 1208 published papers about the domain knowledge modeling. They involve a lot of field: automation technology, book intelligence, digital library, education management, etc. Although there are relevant disciplines (Such as management science and engineering discipline and software engineering discipline) whose knowledge modeling results have published, but the literature indicates that the researches have just 
completed one or a few courses of the holistic knowledge database. A complete and practical domain knowledge system has not been formed. And as for knowledge modeling in the domain of information system for teaching, there are few achievements published.

This paper will review the research on domain knowledge modeling of information system and contains four parts. The first part is the introduction. The second part is the analysis and evaluation of the domain knowledge modeling method based on ontology, which mainly includes the main skeleton method, the seven step method and so on. The third part is the analysis and evaluation of the domain knowledge modeling technology based on ontology, which mainly includes the protégé, the mind map and the other technology. In the end, the contribution of this thesis and the future research directions are pointed out.

\section{THE REPRESENTATION METHODS OF DOMAIN KNOWLEDGE}

There are many ways to express domain knowledge of information system. But because the information system discipline is a highly interdisciplinary subject, it involves many domains such as computer, management, mathematics, economics and accounting and so on. The field involves a wide range, the teaching courses are colorful and toilsome. What's more, the relationship between the curriculum and the curriculum knowledge points are complex and varied. Additionally,due to the different levels of the relevant professional courses in various colleges and universities, the difficulty of knowledge representation in the field of information system is increased. At present, the most commonly used methods of knowledge representation are first order predicate representation, generative representation, frame representation, and representation based on ontology, and their characteristics are listed in Table 1.According to the characteristics of the information system, the analysis of these methods is as follows: The first order predicate representation method has the characteristics of strict general expression and easy implementation, but the efficiency is too low and the flexibility is poor. When knowledge is too large, this method is likely to produce knowledge explosion. At the same time, this method can only be used to express the determined knowledge. But the knowledge of information systems are complex and diverse, and in the process of knowledge representation and modeling, developers want to be able to deal with uncertain knowledge effectively. So the method of first order predicate logic is not practical in the information system domain knowledge representation.

TABLE I. DOMAIN KNOWLEDGE REPRESENTATION METHODS

\begin{tabular}{|c|c|c|c|c|}
\hline Method & Knowledge & $\begin{array}{c}\text { Efficienc } \\
\mathrm{y}\end{array}$ & Maturity & Difficulty \\
\hline $\begin{array}{c}\text { First-order } \\
\text { predicate }\end{array}$ & Determined,simple & lower & mature & Very ease \\
\hline $\begin{array}{c}\text { Generativ } \\
\text { e }\end{array}$ & uncertain & low & maturer & easy \\
\hline Frame & $\begin{array}{l}\text { No process } \\
\text { knowledge }\end{array}$ & higher & maturer & moderate \\
\hline Ontology & $\begin{array}{l}\text { Large quantity, } \\
\text { uncertain,NLP }\end{array}$ & high & $\begin{array}{c}\text { Maturer } \\
\text { AI }\end{array}$ & moderate \\
\hline
\end{tabular}

The prime advantage of the Generative representation is the capability to effectively control the combination explosion problem, separation between knowledge base and reasoning machine make the relationship between the knowledge points clarified clearly and naturally, and this method can effectively control the saturation problem, also the modular is stronger. At the same time, production representation provides the representation for uncertainty for the very first time. This method contains a single form, a simple reasoning, no complicated calculation and it deals with relatively simple problems. But due to the complicated relation between knowledge field in information systems, thus we hope that computers to excavate relatively special hidden relationships between knowledge points, so generative system will not work; Main framework representation is a framework to represent different classes and their properties. It has the characteristics of inheritance, structural, naturalness, but the deficiency is that it does not articulate procedural knowledge, meaning that it can not solve the problem of "how"; As for the method based on ontology, it has become mainly tool for the application of artificial intelligence, it can represent knowledge in formal specifications, process a large amount of data and realize the knowledge reuse, reconstruction and expansion. It has the obvious advantage in natural language processing. And ontology technology is relatively mature, the literature indicates that knowledge representation based on ontology has achieved some achievements in other knowledge representation field. Based on these features, we think that the ontology technology can be well used in the information system domain knowledge representation.

\section{OVERVIEW OF DOMAIN KNOWLEDGE MODELING BASED ON ONTOLOGY}

\section{A. A Brief Introduction to the Domain Knowledge Modeling Method in Information System Based on Ontology}

According to the related literature, the knowledge modeling based on ontology has the following several kinds of methods, as is shown in the following.

TABLE II. THE DOMAIN KNOWLEDGE REPRESENTATION TOOLS(BASED ON ONTOLOGY)

\begin{tabular}{|c|c|c|c|c|}
\hline Methods & Institute & hard & Area & Efficiency \\
\hline Mainframe & $\begin{array}{c}\text { Edinburgh } \\
\text { institute of } \\
\text { AI }\end{array}$ & low & enterprise & low \\
\hline 7-steps & Stanford & high & medical & higher \\
\hline IDEFS & KBSI & high & enterprise & higher \\
\hline TOVE & Toronto,u & low & business & low \\
\hline Menthonology & Madrid,u & high & Periodic table & higher \\
\hline $\begin{array}{c}\text { KACTUS } \\
\text { engineering }\end{array}$ & SPRIT & high & RLC & moderate \\
\hline $\begin{array}{c}\text { Loop } \\
\text { Acquisition }\end{array}$ & & high & DM & high \\
\hline SENSUS & USC & mid & MT & depends \\
\hline
\end{tabular}

As for the some symbols in the table, here are some denotations: (1)AI refers to Artificial Intelligence, u refers to 
university; USC refers to University of Southern California(2)LC refers to reused life cycle, DM refers to domain modeling, MT refers to machine translation.

\section{B. Evaluation of Modeling Method Based on Domain Knowledge of Information System}

First of all, we believe that the main framework in not appropriate for the information system knowledge representation. The main reason is that during the construction of the main framework, the analysis and establishment of domain knowledge and their relationships in the early stage are mainly done by artificial. While information system has a wide range of knowledge, and their relationships are so complex that the workload will be toilsome. Thus this method is not suitable for modeling in information system domain. TOVE method is a kind of knowledge modeling method based on the knowledge representation of the first order predicate. Due to a wide variety representations of information system domain knowledge, this kind of traditional way of knowledge modeling is not taken. The main purpose of the KACTUS engineering method is to achieve a knowledge base reuse in the entire life cycle of ontology modeling. In the context of continuous updating of knowledge, the realization of this goal is not that significant. And as for the SENSUS method, it takes the technology called "rejection" in essence. For domain knowledge modeling of information system, it can not achieve high efficiency and reliable appliance. When it comes to the 7-steps method, Methontology method and the method of loop acquisition, we think they are comparatively mature knowledge modeling methods for the information system domain knowledge modeling. In the process of the research on knowledge modeling of information system, researchers can choose one or some of them assist their researches.

\section{OVERVIEW OF DOMAIN KNOWLEDGE MODELING TECHNOLOGY BASED ON ONTOLOGY}

\section{A. A Brief Introduction to Domain Knowledge Modeling Technology Based on Ontology}

(1)The first class Includes Ontolingua, OntoSaurus, WebOnto, WebODE, etc. Among them, the common ground of the first three tools:they are based on a particular language (Ontolingua based on Ontolingua language, OntoSaurus based on LOOM language, WebOnto based on OCML language). And to some extent, they all support a variety of ontology description language based on AI.

(2)The second class includes Protégé series, OntoEdit, OilEd, etc. The biggest feature of these tools is that they are independent of the specific language, which can import and export many kinds of ontology description language format based on Web (Such as XML, RDF (S), DAML+OIL, etc). Among them, in addition that OilEd is a separate ontology editing tool, all of them possess an integrated ontology development environment or a set of tools. They support most of the activities in the ontology development lifecycle. Also, because they are all based on the structure of the components, it is easy to provide more features by adding new modules, thus they have good ductility.
The series are widely used open source ontology construction tool developed by Java. It can provide a graphical interface development environment, users can use the property structure to describe the ontology hierarchy. At the same time, it can support background database storage, that is, the system files (including text and pictures) and system components can be separated. and it can control the page showing by using Java database connectivity.

(3) Mind mapping technology is a relatively new knowledge modeling technology. It is also called mental map, which is created by the president of the British mind foundation, Buzan Tony. It illustrates the theme at all levels and can be displayed by using the hierarchical graph, which is related to each other.

In the learning process of the learners, at first, the learners learn the knowledge of a subject and their relationship between each other, and through continuous association and reasoning, they can form a complex network connection in the mind ultimately. Then, with the continuous development of the learner's cognitive process, the network structure of the brain is reconstructed and expanded. In this process, the knowledge points are connected much more tightly and will produce new knowledge.

The most direct function of the mind map is representation of these structures. The higher the dimension of the knowledge point of the thinking map representation, the more closely it $\mathrm{s}$ related to the knowledge points, thus the more quickly the problem is solved. The higher the degree of knowledge abstraction, the better universality for knowledge block to solve the problem. $t$ can be said that thinking map is a strategy that can make the tacit knowledge visualized and unstructured knowledge structured, in the end it promotes to solve the problem effectively.

\section{B. Evaluation of Modeling Technology Based on Domain Knowledge of Information System}

We believe that, in general, the Prot é g é software technology is more suitable for the current domain knowledge modeling of information system. There are two reasons:First, the development of Protégé technology is relatively mature (relative to the Ontolingua and the Mind mapping technology). The literature show that there are many successful cases of knowledge modeling in other areas, which provide successful possibility for the new research direction in the field of information system. Secondly, compared to the Ontolingua technology, the technology of Prot égé software is more flexible and the import and export can be implemented easily. For information system, which has all kinds of knowledge and complex concepts, Prot é gé technology has the obvious advantages.

\section{SUMMARY}

The representation of the domain knowledge of information system is the basis of realizing the adaptive learning platform of information system, and it is the key whether the adaptive learning platform could be efficient and quick. In this paper, according to the need of the adaptive learning platform, we 
analyzed and compared the domain knowledge modeling, including the domain knowledge representation methods, domain knowledge modeling methods and modeling tools based on ontology. Hoping that all of these can have reference and guidance for the representation of complex domain knowledge representation, modeling method and application

The field of the information system has the characteristics of interdisciplinary, strong practicality. While, the domain knowledge modeling for teaching is not only based on the theory(such as constructivism, connectivism)of teaching and learning, but on the knowledge network and curriculum arrangement. So the complexity and difficulty of domain knowledge modeling for information system based on adaptive learning will be much more higher. Based on the analysis of this paper, the research directions in the future will include the following contents:(1)Research on domain knowledge visualization of information system for adaptive learning;(2)Research on adaptive learning algorithm based on ontology knowledge base;(3)The evaluation of adaptive learning effect.

\section{ACKNOWLEDGMENTS}

This paper is partially supported by Beijing Language and Culture University (project number: A201310), Ministry of Education and Baidu Company (project number: 2014-B306).

\section{APPENDIX}

Dr. Li Jimei, corresponding author of this paper, is a Professor of Dean of Department of Information Management, College of Information Sciences, Beijing Language and Culture University, China. Her research interests include information systems, data mining, digital business reports.

Chen Anqi, an undergraduate student majored in Information Management and Information System, currently studying in Beijing Language and Culture University.

\section{REFERENCE}

[1] Zhang Jifang. Application and Construction on Teaching Knowledge Based on Ontology[D]. Nanchang: Nanchang University, 2008.12.

[2] Jiang Tingting, Lv Huixin, Wang Jianhua. The Constitution of Field Repository Based on The Relationship of Knowledge Point[J]. Journal of natural science of Harbin Normal University, 2009,25 (1): 48-50.

[3] Chou Baoyan. Research on knowledge modeling based on domain ontology[M]. Shandong: Shandong Normal University, 2009.5.

[4] Zhang Dongmin, Liao Wenhe, Hu Jian. Design knowledge modeling based on Ontology[J]. Journal of South China University of Technology (NATURAL SCIENCE EDITION), 2005,33 (5): 26-31.

[5] Xu Baoxiang, Ye Peihua. Research on the method of knowledge representation [J]. Information science, 2007,25 (5): 690-694.

[6] Hu Wei. Research on the model of educational resource retrieval based on Ontology and construction of its ontology library[M]. Inner Mongolia: Inner Mongolia University, 2014.5.

[7] Liu Chunlei. Research on knowledge modeling method based on Ontology in educational field[M]. Chongqing: Chongqing University, 2005.5.

[8] Tong Hongxia, Xie Shenquan. Research on the relationship of knowledge points in ICAI $[\mathrm{J}]$. Computer engineering and applications, 2004, (1): 77-87.
[9] Wang Yinglin, Wang Weidong, Wang Zongjiang etc. Reconfigurable knowledge management platform based on Ontology[J]. Computer Integrated Manufacturing System -CIMS, 2003, 9 (12): 1136-1144.

[10] Tang Xiaobo, Wei Zhen, Xu Lei. Modeling method of information system based on Ontology[J]. Information Science, 2008,26 (3): 391-395.

[11] Zhang Mei, Hao Jia, Yan Yan etc. Knowledge modeling technology based on Ontology[J]. Journal of Beijing Institute of Technology, 2010,30 (12): 1405-1410.

[12] Liu Zhiyong. Research on self adaptive learning method and its application based on Ontology[D]. Jilin: Jilin University, 2010.6.

[13] Lin Peiguang, Xu Ruzhi, Yu Zhengtao. Uncertain Knowledge representation based on ontology and cloud theory[J]. Computer engineering and applications, 2008,44 (5): 51-55.

[14] Hu Wenbin, Li Qianmu, Zhang Hong. Model integration of uncertainty based on domain knowledge[J]. Journal of Nanjing University of Science and Technology (NATURAL SCIENCE EDITION), 2010,34 (4): 410-415.

[15] Xu Yi, Zhan Hongfei, Yu Junhe etc. Knowledge modeling method based on specific domain modeling meta model 2012,30, [J] (2): 75-80.

[16] Hu Yiwen, Wu Di, Chen Shaodong. Research on the construction of the knowledge block based on the mind map in the teaching $[\mathrm{J}]$. Science and Technology Square, 2011, (7): 244-248.

[17] Pan Xuwei, Gu Xinjian, Chou Yuanfu, etc. knowledge modeling technology oriented to knowledge management [J]. Computer Integrated Manufacturing System -CIMS, 2003, 9 (7): 518-523.

[18] Nian Zhigang, Liang Shi, Ma Fanglan etc. Research and application of knowledge representation[J]. Computer application research, 2007,24 (5): 234-238.

[19] Jia Wei Qu. Research on the relationship of knowledge points in the network course based on knowledge representation [J]. Continuing education research, 2008, (5): 63-65.

[20] Ma Deng. Ontology construction and application of knowledge point Library in software engineering [D]. Wuhan: Wuhan University of Technology, 2014.4.

[21] Li Gang. Research on the personalized teaching system based on knowledge points and related associations -- Taking $C$ language program design course as an example[D]. Northwestern University, 2011.5.

[22] WELLSBH.A Multi-dimensional Hierarchal Engineering Competency Model Framework[C].Proceedings of 2nd Annual IEEE Systems Conference,2008:1-6.

[23] KAYJ, ontologies for reusable and scrutable student model[C].Proceedings of AIED99 Workshop on Ontology for Intelligent Educational Systems,1999.

[24] Jie Zhang,Bruce Cheung,Lucas Hui.A design of an intelligent tutoring system, TR-2001-01[R].Hong Kong: University of Hong Kong,2001:120. 